\title{
Zinc-responsive acral hyperkeratosis as a mimicker of cutaneous tuberculosis
}

\author{
Vikash Paudel, Setu Mittal, Richa Tripathi, Rupa Thakur, Manish Bhakta Pradhan, \\ Buddhi Raj Pandey
}

Department of Dermatology and Venereology, National Medical College, Bhediyahi-15, Birgunj, Parsa, Province 2, Nepal

Corresponding author: Vikash Paudel, MD, E-mail: vikashpoudel@iom.edu.np

\begin{abstract}
Zinc-responsive acral hyperkeratosis is regarded as a novel entity masquerading numerous skin disorders, such as psoriasis, acral necrolytic erythema, and tuberculosis. Tuberculosis itself is a great imitator and so is its cutaneous form. Herein, we present the case of a female misdiagnosed as a case of tuberculosis verrucosa cutis due to clinical, biochemical, and histopathological features. The patient completed a full course of anti-tubercular therapy without an improvement and showed a dramatic response after a therapeutic trial of oral zinc. Thus, the patient was diagnosed as a case of zinc-responsive acral hyperkeratosis. In any form of acral hyperkeratotic lesions, zinc-responsive acral hyperkeratosis must be considered as a differential diagnosis.
\end{abstract}

Key words: Acral hyperkeratosis; Cutaneous tuberculosis; Nepal; Tuberculosis verrucosa cutis; Zinc

\section{INTRODUCTION}

Zinc-responsive acral hyperkeratosis $(\mathrm{ZRAH})$ is a rare dermatological disorder recently announced as a novel entity, which clinically presents itself as acral hyperkeratosis and shows a dramatic response to zinc [1]. Cutaneous tuberculosis (CTB) is uncommon and varies in its clinical manifestations depending on the host's immune status and is also a great classic imitator. Even with diagnostic advancements, misdiagnosis and undertreatment are likely, and consideration of this particular problem is recommended [2]. Herein, we present a complex case of a novel tuberculosis imitator: ZRAH in an adolescent female with clinical and pathological misdiagnosis of cutaneous tuberculosis of the bilateral foot cured with a dramatical response with a micronutrient for growth and development, elemental oral zinc. To our knowledge, this is the first case report of zinc-responsive acral hyperkeratosis from Nepal.

\section{CASE REPORT}

This is a case of an adolescent female with skin lesions in the dorsum of the bilateral feet present for one year. The patient had hyperpigmented and hyperkeratotic skin plaques ranging in size from $5 \times$ $5 \mathrm{~cm}$ to $5 \times 7 \mathrm{~cm}$ in the dorsum of the foot, ankle, and tendinous-calcaneus region up to the distal lower leg (Figs. la and 1b). The patient underwent multiple biopsies with multiple treatment modalities in multiple clinical settings before being admitted to our hospital. Our initial differential diagnoses were tuberculosis verrucosa cutis, acral necrolytic erythema, and psoriasis. Biochemical (high ESR and Monteux at $15 \mathrm{~cm}$ in 72 hours) and clinical parameters and histopathological findings with a chronic lymphocytic infiltrate, with Langerhans cells and epithelioid cells in the dermis, were supportive of tuberculosis verrucosa cutis (TVC). On serological screening, the patient was negative for HIV, hepatitis $\mathrm{B}$, and hepatitis $\mathrm{C}$ and non-reactive for the venereal disease research laboratory (VDRL) test. Because of the high prevalence of tuberculosis in the community and other supportive parameters, a therapeutic trial of anti-tubercular therapy (ATT) was initiated along with keratolytics agents such as salicylic acid and emollients. The patient was put under conventional antitubercular therapy (ATT) for six months, including two months of the intensive phase and a continuation

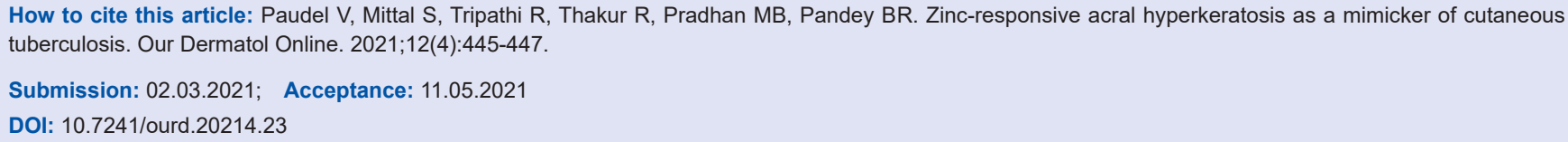


phase of four months. Even after the standard antitubercular regimen administered for nearly six months, she had no significant improvements in the lesions, which were present on the legs. As CTB is an imitator of numerous clinical entities, an alternative diagnosis was considered. Regarding the clinical features of the bilateral hyperkeratotic lesions and acral involvement, a presumptive diagnosis of zincresponsive acral dermatosis was considered and the patient was put under a therapeutic trial of a high dose of zinc sulfate $(300 \mathrm{mg} / \mathrm{day})$. To our surprise, she improved dramatically in two weeks of therapy and was almost cured in two months (Figs. 2a and 2b) Thus, a clinicohistopathological mimicker of cutaneous tuberculosis was treated and cured with oral zinc therapy and a retrospective diagnosis of zincresponsive acral hyperkeratosis was reached. Figure 3 shows the timeline progression of the disease.

\section{DISCUSSION}

Acral hyperkeratosis may be present in various skin diseases, such as acral necrolytic erythema, psoriasis, lichen simplex chronicus, verrucous tuberculosis, and ZRAH [1]. Acral necrolytic erythema has a clinical presentation similar to ZRAH but the response to zinc is not very dramatic and is usually associated with viral hepatitis $\mathrm{C}$ infection. Although the use of zinc in dermatology has been suggested for numerous skin diseases, its role in cutaneous tuberculosis is not discussed in the literature [3]. Clinically and histologically, lichen simplex chronicus and psoriasis vulgaris outweighed the diagnosis in our case. $\mathrm{CTB}$, which is caused by the invasion of the skin by Mycobacterium tuberculosis or Mycobacterium bovis, or following a BCG vaccination, may be present in various forms. It is a less common form and only accounts for about $1 \%$ to $2 \%$ of all extrapulmonary cases [4]. Tuberculosis verrucosa cutis, a type of CTB, occurs after direct inoculation into the skin and presents as a brownish-red warty growth most often in the feet, buttocks, and hands. Because it has various clinical and histopathological mimickers, diagnosis and treatment become a challenge, even with diagnostic and therapeutic advancements [2,5]. The patient's clinical presentation of tuberculosis also varies with immune status, with patients with HIV infection possessing even more bizarre presentations [6]. There is suggested evidence of a change in serum zinc levels in tuberculosis but no proven evidence as diagnostic or therapeutic markers [7]. Our patient

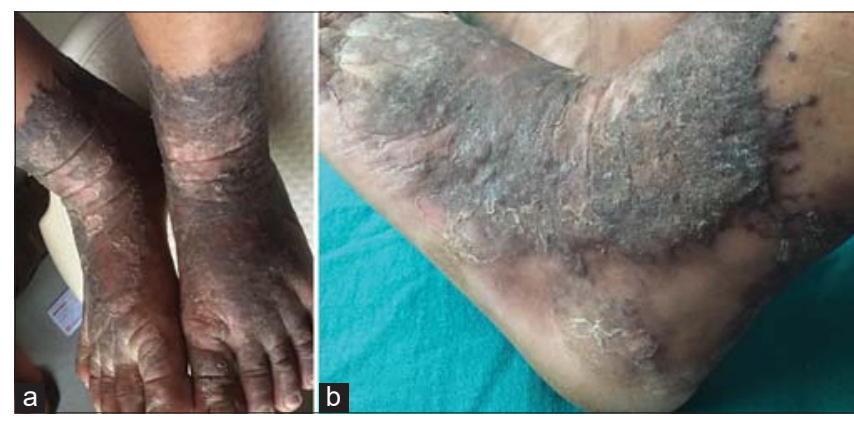

Figure 1: (a) Anterior view of the lesion before the treatment. (b) Lateral view of the lesions before the treatment.

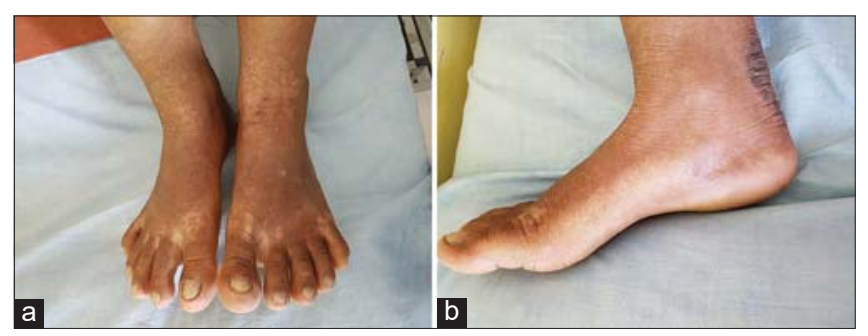

Figure 2: (a) Complete resolution after the zinc therapy. (b) Complete resolution after the zinc therapy.

presented with multiple acral warty lesions over the bilateral dorsal foot with positive Manteaux, a high ESR, and histology suggestive of TVC. However, the poor response to ATT led to us revise our diagnosis and consider a therapeutic trial of high-dose zinc. There was a dramatic response to the zinc therapy with almost complete clearance of the lesions. The additional high dose zinc therapy would not only be supplemental to ATT, as there was no change in ATT for almost six months and a dramatic response was achieved with zinc therapy. There have been reports of acral plaques and significant response to oral zinc dilemmas in nomenclature as novel entities or subsets of well-known dermatoses [1].

\section{CONCLUSION}

Clinicohistologically diagnosed cases of verrucous cutaneous tuberculosis, if non-responsive to conventional therapy, require a high suspicion of mimickers as zinc-responsive acral dermatoses may mimic the hyperkeratotic variant of CTB.

\section{Consent}

The examination of the patient was conducted according to the principles of the Declaration of Helsinki.

The authors certify that they have obtained all appropriate patient consent forms, in which the patients gave their consent for images 


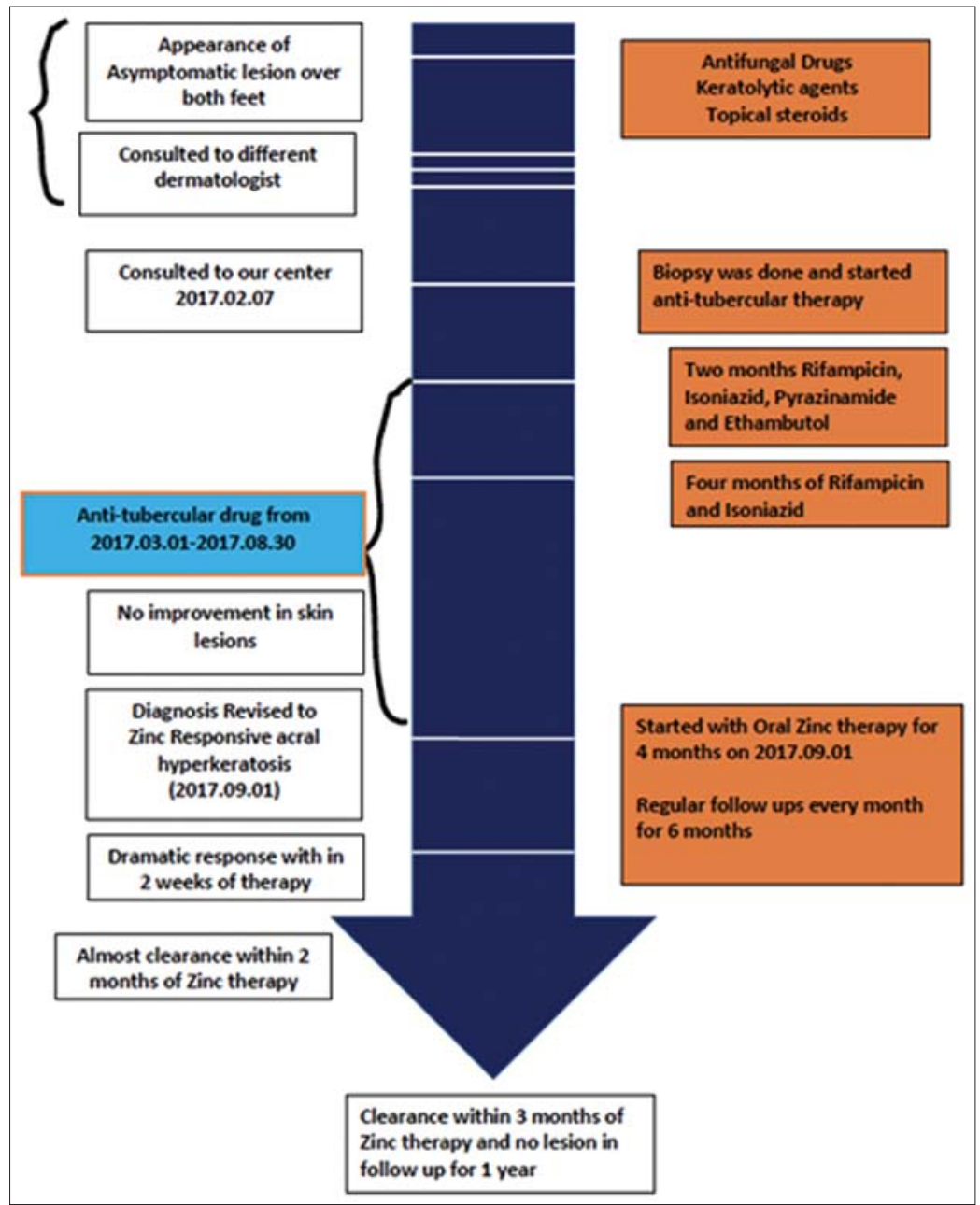

Figure 3: Progression timeline of the disease and its clearance.

and other clinical information to be included in the journal. The patients understand that their names and initials will not be published and due effort will be made to conceal their identity, but that anonymity cannot be guaranteed.

\section{REFERENCES}

1. Ghosh A, Aggarwal I, De A, Samanta A, Chatterjee G, Bala S, et al. Zinc-responsive acral hyperkeratotic dermatosis: A novel entity or a subset of some well-known dermatosis? Indian J Dermatol. 2015;60:136-41.

2. Chen Q, Chen W, Hao F. Cutaneous tuberculosis: A great imitator. Clin Dermatol. 2019;37:192-9.

3. Gupta M, Mahajan VK, Mehta KS, Chauhan PS. Zinc therapy in dermatology: A review. Dermatol Res Pract. 2014;2014:709152.
4. Santos JB, Figueiredo AR, Ferraz CE, Oliveira MH, Silva PG, Medeiros VL. Cutaneous tuberculosis: epidemiologic, etiopathogenic and clinical aspects - Part I. An Bras Dermatol. 2014;89:219-28.

5. Manjumeena D, Sundaramoorthy S. Tuberculosis verrucosa cutis masqerading as chromoblastomycosis - A case report. Our Dermatol Online. 2018;9:275-8.

6. Paudel V, Chudal D, Pokhrel DB. Tuberculosis and HIV coinfection; the deadly duos in vulva. Indian J Tuberc. 2018;65:277-9.

7. Gupta KB, Gupta R, Atreja A, Verma M, Vishvkarma S. Tuberculosis and nutrition. Lung India. 2009;26:9-16.

Copyright by Vikash Paudel, et al. This is an open access article distributed under the terms of the Creative Commons Attribution License which permits unrestricted use, distribution, and reproduction in any medium, provided the original author and source are credited.

Source of Support: Nil, Conflict of Interest: None declared. 